\title{
Effect of PTEN loss on metabolic reprogramming in prostate cancer cells
}

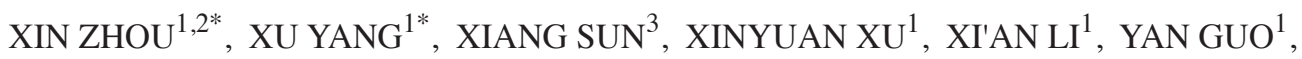 \\ JIANCAI WANG ${ }^{1}$, XIA LI $^{1}$, LIBO YAO $^{1}$, HE WANG $^{1}$ and LAN SHEN ${ }^{1}$
}

\begin{abstract}
${ }^{1}$ The State Key Laboratory of Cancer Biology, Department of Biochemistry and Molecular Biology, The Fourth Military Medical University; ${ }^{2}$ Department of Urology, Tangdu Hospital, The Fourth Military Medical University; ${ }^{3}$ Department of Prosthodontics, School of Stomatology, The Fourth Military Medical University, Xi'an, Shaanxi 710032, P.R. China
\end{abstract}

Received April 28,2018; Accepted December 3, 2018

DOI: $10.3892 / 01.2019 .9932$

\begin{abstract}
The tumor suppressor gene PTEN is one of the most often deleted genes in human prostate cancer. Loss of PTEN is an important event in prostate carcinogenesis. Metabolic reprogramming induced by PTEN loss fuels malignant growth and proliferation of prostate cancer cells. Targeted metabolomics analysis was used to investigate the effects of PTEN loss on intracellular metabolic pathways in prostate cancer cells. DU-145 cells were transfected with PTEN siRNAs (siRNA-1 and siRNA-2) for $48 \mathrm{~h}$, and endogenous PTEN expression was monitored by western blotting. Changes in intracellular metabolites were determined by liquid chromatographytandem mass chromatography (LC-MS/MS) and gas chromatography-mass spectrometry (GC-MS). Most intracellular metabolites involved in glycolysis and glutaminolysis were increased in PTEN knockdown prostate cancer cells. In addition, most intracellular metabolites involved in fatty acid de novo synthesis, fatty acid beta oxidation and branched chain amino acid catabolism were also increased in PTEN knockdown prostate cancer cells. These results revealed that PTEN loss induced the metabolic reprogramming of prostate cancer cells and promoted the malignant proliferation of prostate cancer cells. The present metabolomics analysis indicates that tumor suppressor gene PTEN mutation or deletion can induce metabolic reprogramming in prostate cancer cells and tumorigenesis by altering the metabolic flux of glycolysis, glutaminolysis, fatty acid metabolism and branched chain
\end{abstract}

Correspondence to: Dr Lan Shen or Dr He Wang, The State Key Laboratory of Cancer Biology, Department of Biochemistry and Molecular Biology, The Fourth Military Medical University, 169 Changlexi Road, Xi'an, Shaanxi 710032, P.R. China

E-mail: lanshen@fmmu.edu.cn

E-mail: hewang@fmmu.edu.cn

*Contributed equally

Key words: tumor suppressor gene, PTEN, glycolysis, glutaminolysis, metabolomics amino acid catabolism pathways. Metabolic reprogramming is one of the contributors to PTEN-loss driven prostate cancer.

\section{Introduction}

The tumor suppressor gene PTEN is frequently deleted or mutated in human prostate cancer (1). Prostate-specific PTEN knockout in transgenic mice was revealed to promote prostate cancer progression and metastasis (2). PTEN encodes a dual-specificity protein phosphatase and negatively regulates the phosphatidylinositol 3-kinase (PI3K)/AKT signaling pathway. Loss of PTEN leads to the activation of the PI3K/Akt signaling pathway and tumorigenesis (3). Furthermore, PTEN can interact directly with p53 and regulate p53 stability and transcriptional activity in an Akt-independent manner $(4,5)$. In addition, PTEN loss can also lead to the activation of the c-Jun $\mathrm{NH}_{2}$-terminal kinase (JNK) pathway independent of the PI3K/Akt signaling pathway (6). Therefore, PTEN loss changes several pathways to promote prostate cancer development.

Different from well-differentiated tissue cells, which mainly depend on the energy supplied by mitochondrial oxidative phosphorylation to keep cellular activities, tumor cells prefer aerobic glycolysis. This preference generates energy by converting glucose to lactate in an aerobic environment as the main way to supply ATP for their proliferation and other cellular processes. This paradox phenomenon is named the Warburg effect (7). Compared with other epithelial cancers, there are more changes in lipid metabolism of prostate cancers $(8,9)$. PTEN loss can lead to the overexpression of fatty acid synthase (FAS), which is associated closely with both cholesterol and fatty acid biosynthesis in human tumors, especially in prostate cancer (10). In addition, the transgenic mouse model with global PTEN overexpression resulted in reduced glucose and glutamine uptake, increased mitochondrial oxidative phosphorylation, and was resistant to oncogenic transformation (11). The inhibition of the metabolic reprogramming induced by PTEN loss is possibly a potential target for cancer prevention and therapy.

In the present study, the metabolic reprogramming in siRNA-mediated PTEN knockdown prostate cancer cells DU-145 was examined with a novel comprehensive metabolomics analysis method. Comprehensive metabolic analysis 
is a breakthrough method capable of clarifying the global profiles of abundant metabolites. The glucose, glutamine and fatty acid metabolic pathways were focused on, and the related metabolic changes induced by PTEN loss in DU-145 cells were reported.

\section{Materials and methods}

Cell culture and treatment with siRNAs. DU-145 and 22Rv1 cells were purchased from the American Type Culture Collection (ATCC; Manassas, VA). DU-145 cells were cultured in Gibco DMEM (Thermo Fisher Scientific, Inc., Waltham, MA, USA) and 22Rv1 cells were cultured in Gibco RPMI-1640 (Thermo Fisher Scientific, Inc.) containing 10\% fetal calf serum, $100 \mathrm{U} / \mathrm{ml}$ penicillin and $100 \mu \mathrm{g} / \mathrm{ml}$ streptomycin in a humidified atmosphere containing $5 \% \mathrm{CO}_{2}$ set at $37^{\circ} \mathrm{C}$. Every two days, media were changed to provide cells with optimal growth conditions.

All siRNA oligonucleotides were purchased from GenePharma Co., Ltd. (Shanghai, China). The sequences of all specific siRNAs targeted to human PTEN are displayed in Fig. 1A. The siRNAs were transfected into cells with Lipofectamine 2000 (Invitrogen; Thermo Fisher Scientific, Inc.). In the small interfering RNA (siRNA) data set, prostate cancer cells were divided into 3 groups. The control group was transfected with control siRNA at a dose of $40 \mathrm{nM}$. The experimental group was transfected with either PTEN siRNA-1 or PTEN siRNA-2 at a dose of 20 or $40 \mathrm{nM}$. The media of the different groups were replaced by the fresh complete culture media after transfection for $6 \mathrm{~h}$. Recombinant lentiviral vectors were constructed with an Invitrogen ViraPower ${ }^{\mathrm{TM}}$ Lentiviral System (Thermo Fisher Scientific, Inc.) in our laboratory. The lentiviral vectors pLKO-Scramble/PTEN shRNA-1/PTEN shRNA-2, PAX2 and PMD2G were transfected into 293T cells using Lipofectamine 2000 (Invitrogen; Thermo Fisher Scientific, Inc.) according to the manufacturer's instructions. DU-145 and 22Rv1 cells were infected with the viral medium from $293 \mathrm{~T}$ cells $48 \mathrm{~h}$ after transfection.

Quantitative real-time PCR. After transfection with siRNAs for $48 \mathrm{~h}$, total RNA was isolated from cells using TRIzol Reagent (Invitrogen; Thermo Fisher Scientific, Inc.), and then complementary DNA (cDNA) was synthesized using AMV reverse transcriptase (Promega Corporation, Madison, WI, USA) according to the manufacturer's instructions. The cDNA was used as a template for quantitative real-time PCR using an ABI Prism 7500 real-time PCR instrument (Applied Biosystems; Thermo Fisher Scientific, Inc.). The primer sequences for PTEN and $\beta$-actin were as follows: PTEN forward primer, 5'-CGACGGGAAGACAAGTTCAT-3' and reverse, 5'-AGG TTTCCTCTGGTCCTGGT-3'; $\beta$-actin forward primer, 5'-CGCGAGAAGATGACCCAGAT-3' and reverse, 5'-GTA CGGCCAGAGGCGTACAG-3'. The following thermocycling conditions were maintained: $95^{\circ} \mathrm{C}$ for $3 \mathrm{~min} ; 95^{\circ} \mathrm{C}$ for $10 \mathrm{sec}$ and $58^{\circ} \mathrm{C}$ for $30 \mathrm{sec}$ for 39 cycles; and melting curve analysis using an increase from 65.0 to $95.0^{\circ} \mathrm{C}$ in $0.5^{\circ} \mathrm{C}$ increments for $5 \mathrm{sec}$. Independent experiments were repeated three times. The relative expression levels of mRNA were analyzed using Bio-Rad CFX Manager v3.1 software (Bio-Rad Laboratories, Inc., Hercules, CA, USA) with the $2^{-\Delta \Delta C q}$ method (12).
Immunoblot analysis. After transfection with siRNAs for $48 \mathrm{~h}$, cells were lysed in cell lysis buffer containing $20 \mathrm{mM}$ Tris- $\mathrm{HCl}, 0.5 \mathrm{M} \mathrm{NaCl}, 0.25 \%$ Triton $\mathrm{X}-100,1 \mathrm{mM}$ EDTA, 1 mM EGTA, $10 \mathrm{mM} \beta$-glycophosphate, $300 \mu \mathrm{M}$ $\mathrm{Na}_{3} \mathrm{VO}_{4}, 10 \mathrm{mM} \mathrm{NaF}, 1 \mathrm{mM}$ benzamidine, $2 \mu \mathrm{M}$ PMSF and $1 \mathrm{mM}$ DTT. Protein concentrations were determined by a BCA protein assay (Thermo Fisher Scientific, Inc.). Proteins were subjected to $10 \%$ SDS-PAGE, transferred on to nitrocellulose membranes, blocked in 5\% BSA, and followed by washing with TBS with Tween-20. The blots were then incubated with primary antibodies for $12 \mathrm{~h}$ at $4^{\circ} \mathrm{C}$ followed by incubation with the secondary antibody for $2 \mathrm{~h}$ at room temperature. The following primary antibodies were used: polyclonal rabbit anti-human phospho-Akt (Ser473) (dilution, 1:1,000; cat. no. 9271S), polyclonal rabbit anti-human Akt (dilution, 1:1,000; cat. no. 9272S), polyclonal rabbit anti-human PTEN (dilution, 1:1,000; cat. no. 9552S), polyclonal rabbit anti-human HK1 (dilution, 1:1,000; cat. no. $2024 \mathrm{~S}$ ), polyclonal rabbit anti-human HK2 (dilution, 1:1,000; cat. no. 2106S), polyclonal rabbit anti-human PKM1/2 (dilution, 1:1,000; cat. no. 3186S), polyclonal rabbit anti-human PKM2 (dilution, 1:1,000; cat. no. 3198S), polyclonal rabbit anti-human LDHA (dilution, 1:1,000; cat. no. 2012S), polyclonal rabbit anti-human PDH (dilution, 1:1,000; cat. no. 3205S), polyclonal rabbit anti-human PFKP (dilution, 1:1,000; cat. no. 5412S) all from Cell Signaling Technology, Inc. (Danvers, MA, USA), and monoclonal rabbit anti-human PCB (dilution, 1:2,000; cat. no. ab126707; Abcam, Cambridge, UK), or polyclonal rabbit anti-human $\beta$-actin (dilution, 1:1,000; cat. no. 4970S; Cell Signaling Technology, Inc.). Membranes were then incubated with horesradish peroxidase-conjugated secondary antibody polyclonal goat anti-rabbit IgG (dilution, 1:3,000; cat. no. ab97051; Abcam) for $1 \mathrm{~h}$ and visualized with SuperSignal ${ }^{\mathrm{TM}}$ West Femto Maximum Sensitivity Substrate (Thermo Fisher Scientific, Inc.) according to the manufacturer's recommended protocol. Quantification was performed using Image $\mathrm{J}$ software version $1.45 \mathrm{~S}$ (National institutes of Health, Bethesda, MD, USA).

Glucose consumption and lactate production. After $48 \mathrm{~h}$ of transfection, all culture media were collected for glucose consumption and lactate production analysis. The concentrations of glucose in culture media were assessed after $24 \mathrm{~h}$ using a Glucose Detection Kit (Invitrogen; Thermo Fisher Scientific, Inc.). Briefly, the culture media was mixed with the colorimetric substrate and horseradish peroxidase and the reaction was initiated by the addition of glucose oxidase in a 96-well plate. The reaction was incubated for $30 \mathrm{~min}$ at room temperature and the product was assessed by a microplate reader at an absorbance of $560 \mathrm{~nm}$. Glucose consumption was the difference of the glucose concentration between the spent medium and the unused medium. The concentrations of lactate in the culture media were assessed using a Lactate Assay Kit (Jiancheng Bioengineering, Nanjing, China). Briefly, the culture media was mixed with lactate assay buffer in a 96-well plate. Then reaction buffer was added to every well and incubated for $30 \mathrm{~min}$ at room temperature. The lactate production was assessed by a microplate reader at an absorbance of $570 \mathrm{~nm}$. 
MTT assay. Cells infected with lentivirus containing PTEN shRNA or control shRNA were seeded into 96-well plates in triplicate at a starting density of $1 \times 10^{4}$ cells/well. Treated cells were washed and incubated with tetrazolium salt (MTT, $100 \mu \mathrm{g} / \mathrm{ml}$; Sigma-Aldrich; Merck KGaA, Darmstadt, Germany) at $37^{\circ} \mathrm{C}$ for $4 \mathrm{~h}$. The supernatant was removed, and $150 \mu \mathrm{l}$ of dimethyl sulfoxide (DMSO) was added to each well. The absorbance (OD) of the reaction solution at $490 \mathrm{~nm}$ was recorded.

\section{Metabolomics analysis of organic acid}

Sample preparation for metabolic profiling. The siRNA-treated cells were washed twice with room temperature D-Hank's solution, digested with trypsin for 5 min and then collected in a culture tube. The collected solution was centrifuged $\left(4^{\circ} \mathrm{C}, 380 \mathrm{x} \mathrm{g}, 2 \mathrm{~min}\right)$, and the supernatant was discarded. Next, $500 \mu \mathrm{l}$ of methyl alcohol $\left(4^{\circ} \mathrm{C}\right)$ was added to the tube containing the cell pellet and was vortexed for $30 \mathrm{sec}$. After three freeze-thaw cycles, the mixture was centrifuged $\left(4^{\circ} \mathrm{C}\right.$, $15,000 \mathrm{xg}, 10 \mathrm{~min})$. The upper layer was used for organic acid and fatty acid metabolomics analysis.

For organic acid analysis, the supernatant was placed in a $1.5 \mathrm{ml}$ polypropylene centrifuge tube, then $25 \mu \mathrm{l}$ each of margarate (MGA) and tetracosane (C24) and $40 \mathrm{mg}$ of tropate (TA) were added as internal standards. Subsequently, $50 \mu \mathrm{l}$ of hydroxylamine hydrochloride, sulfuric acid and saturated ammonium chloride were added to the fouling reaction. The the mixed solution was extracted twice by ether. After centrifugation for $5 \mathrm{~min}$, the supernatant was placed in a $1.5 \mathrm{ml}$ vial. The aliquots were evaporated to dryness in a vacuum concentrator. The final dry residue, $110 \mu \mathrm{l}$ of a mixture of BSTFA and TMCS (10:1, v:v) were added and dissolved. The solution was then transferred to a 1-ml screw top vial, tightly capped and trimethylsilyl (TMS)-derivatized at $80^{\circ} \mathrm{C}$ for $30 \mathrm{~min}$. The samples were then ready for GC-MS analysis.

Mass spectrometry. Samples were analyzed using a Gas Chromatography-Mass Spectrometer (GC-MS, 7890-5975; Agilent Technologies, Inc., Santa Clara, CA, USA). The capillary column was a fused-silica DB-5 column (30 m x $0.25 \mathrm{~mm}$ i.d.) with a $0.25-\mu \mathrm{m}$ film thickness of $5 \%$ phenylmethylsilicone. Mass spectra were obtained by standard electron impact ionization scanning from $\mathrm{m} / \mathrm{z} 50$ to $\mathrm{m} / \mathrm{z} 750$ at a rate of $0.35 \mathrm{sec} / \mathrm{cycle}$. The temperature program started at $150^{\circ} \mathrm{C}$ with an initial hold for $1 \mathrm{~min}$, and the temperature was then increased at a rate of $10^{\circ} \mathrm{C} / \mathrm{min}$ to $285^{\circ} \mathrm{C}$ with a final hold for $8 \mathrm{~min}$. The temperatures of the injection port and transfer line were both $280^{\circ} \mathrm{C}$. The flow rate of the helium carrier was $1.5 \mathrm{ml} / \mathrm{min}$, and the linear velocity was $38.5 \mathrm{~m} / \mathrm{sec}$. One $\mathrm{ml}$ of the final derivatized aliquot was injected into the GC-MS and analyzed in the split mode (10:1).

GC-MS data processing and identification of the metabolites. The compounds were identified by automatically comparing the MS spectra, in-source fragments, and ion features of each peak in the experimental samples with those of reference standards or those available in libraries, such as Mainlib and Publib in the National Institute of Standards and Technology (NIST) library 2.0 (2012) or Wiley 9 (Wiley-VCH Verlag $\mathrm{GmbH} \&$ Co. KGaA, Weinheim, Germany).
Metabolomics analysis of fatty acid

Sample preparation for metabolic profiling. For fatty acid analysis, the supernatant was placed in a polypropylene microtiter plate. Methanolic internal standard solution (150 $\mu \mathrm{l})$ was added manually. The microtiter plate was gently shaken during the $30 \mathrm{~min}$ extraction of acyl-carnitine markers. The methanol extract was then manually transferred to a second polypropylene microtiter plate and dried by a Pressure Blowing Concentrator at $50^{\circ} \mathrm{C}$. Butanol- $\mathrm{HCl}(60 \mu \mathrm{l})$ was manually placed in each sample well and the microtiter plate was covered with a thin Teflon sheet under a heavy weight and placed at $70^{\circ} \mathrm{C}$ in a forced air oven for $15 \mathrm{~min}$. After the plate was removed from the oven, the Pressure Blowing Concentrator removed the remaining butanol-HCl. The butanol-derivatized samples were reconstituted with $100 \mu 1$ of acetonitrile and water (70:30 by volume), and each plate was covered with aluminum foil. The samples were then ready for LC-MS analysis.

Mass spectrometry. Samples were analyzed using liquid chromatograph-tandem mass spectrum (LC20AD; Shimadzu, Kyoto, Japan; API 4000+; AB-Sciex, Framingham, MA, USA).

$L C$. A mixture of acetonitrile and water $(70: 30, \mathrm{v} / \mathrm{v})$ was used as carrier solution at a flow rate of $0.14 \mathrm{ml} / \mathrm{min}$. A step gradient program was developed for the best separation of amino acids: $0.14 \mathrm{ml} / \mathrm{min}, 0.2 \mathrm{~min} ; 0.03 \mathrm{ml} / \mathrm{min}, 1 \mathrm{~min} ; 0.3 \mathrm{ml} / \mathrm{min}$, $0.2 \mathrm{~min}$. An $18-\mu \mathrm{l}$ sample was injected into the carrier solution. Then, the stream was introduced to the ion source for MS/MS without column separation.

Mass spectrometer. The mass spectrum (MS) parameters in positive ion mode (ESI + MODE) were as follows: Heater Temp, $300^{\circ} \mathrm{C}$; Capillary Temp, $350^{\circ} \mathrm{C}$; Sheath Gas Flow rate, 45 arb; Aux Gas Flow Rate, 15 arb; Sweep Gas Flow Rate, $1 \mathrm{arb}$; Spray voltage, 3.0 KV. Product Ion Scan modes were defined to analyze fatty acid metabolism. All data acquisition and processing were carried out using Analyst 1.5.2 software (AB-Sciex).

Statistical Analysis. Statistical analysis was performed using SPSS 17.0 software (SPSS, Inc., Chicago, IL, USA). The chi-square test was used to evaluate the significance of the differences in the cell line samples. Statistical significance was assumed at a threshold of $\mathrm{P}<0.05$. Metabolic pathway analysis and enrichment analysis were performed by MBROLE 2.0 (http://csbg.cnb.csic.es/mbrole2/) and KEGGgraph (http://www. bioconductor.org/packages/2.4/bioc/html/KEGGgraph.html).

The function of KEGGgraph suggested that $>$ library (KEGG.db), > tmp <- tempfile, > pName <- 'Phe signaling pathway', > pId <- mget (pName, KEGGPATHNAME2ID), $>$ retrieveKGML(pId, organism='cel', destfile $=$ tmp, method='wget', quiet=TRUE).

\section{Results}

Enhanced glucose consumption and lactate production. To investigate the metabolic changes in PTEN knockdown prostate cancer, we synthesized two pairs of PTEN siRNAs and a control siRNA (Fig. 1A). After the prostate cancer cell line DU-145 was transfected with 20 or $40 \mathrm{nM}$ siRNAs for 
A

Control siRNA $5^{\prime}$ to $3^{\prime}$ :
UUCUCCGAACGUGUCACGUTT ACGUGACACGUUCGGAGA.ATT

PTEN siRNAI 5" to 3" :

GAUCAGCAUACACAAAUUATT UAAUUUGUGUAUGCUGAUCTT

PTEN siRNA2 $5{ }^{\circ}$ to $3^{\prime}$ :

ACAAAUGAAGGGAUAUAAATT UUUAUAUCCCUUCAUUUGUGG
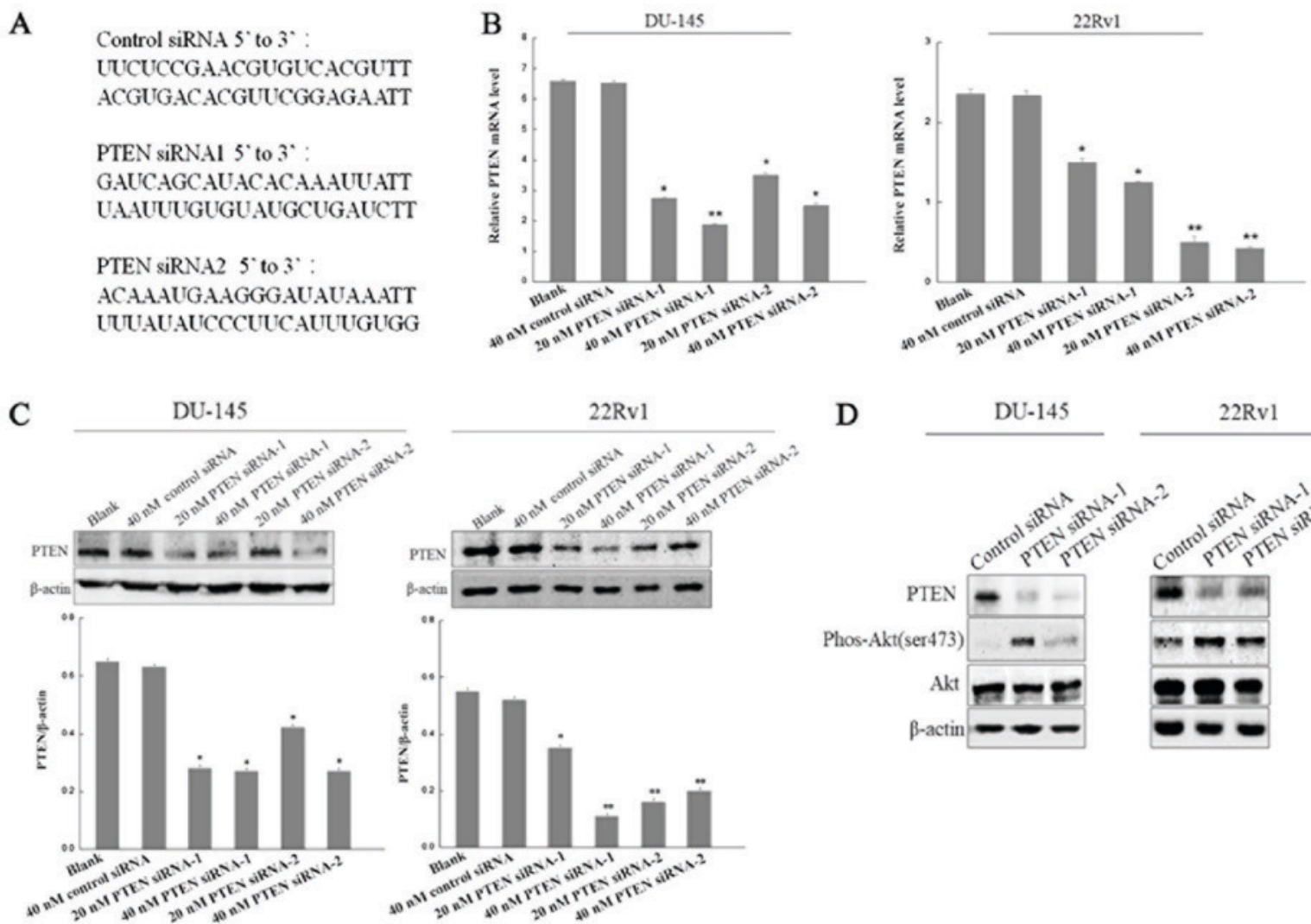

D
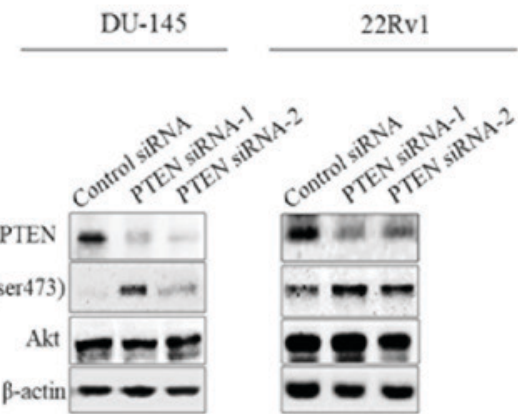
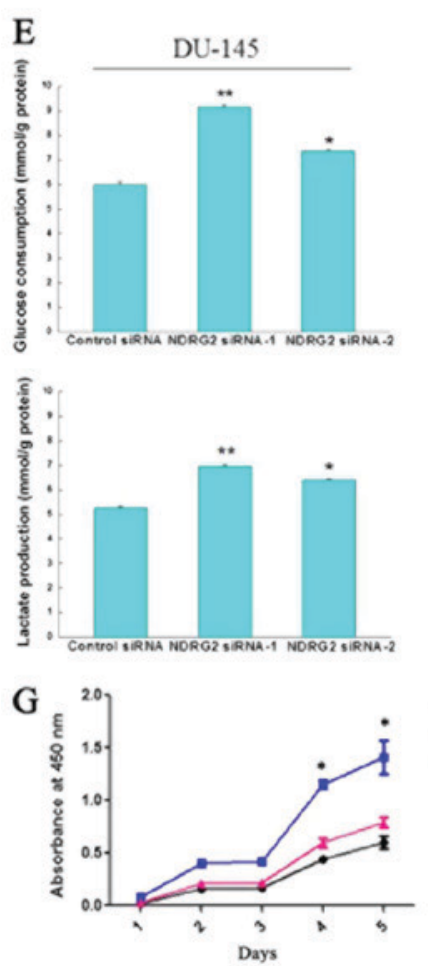

F
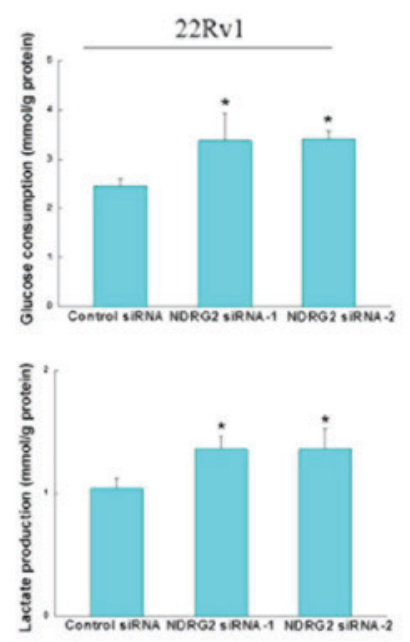

-DU-145 control shRNA -DU-145 PTEN shRNA-1
क- DU-145 PTEN shRNA-2
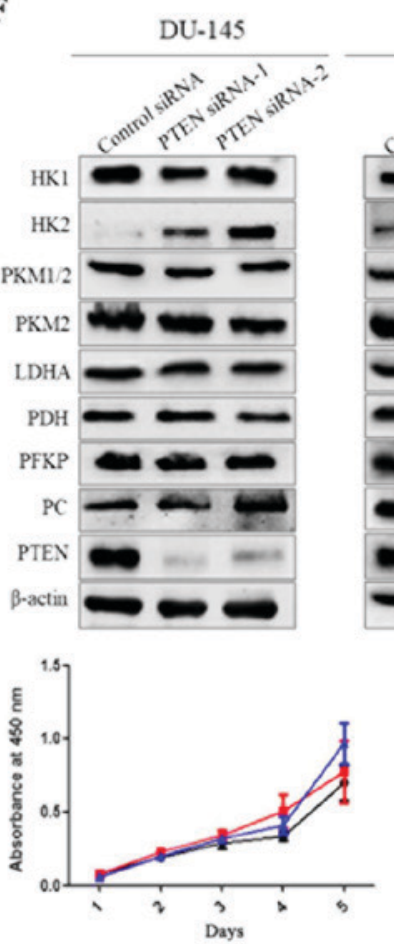

22Rv1

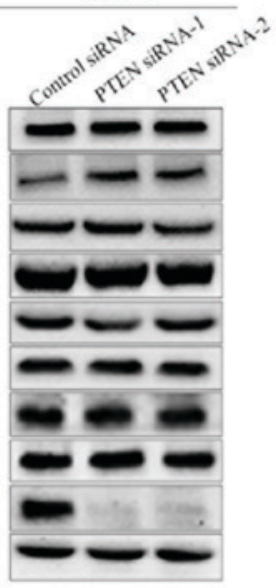

- 22 Rvl control shRNA

- 22Rvi PTEN shRNA-1

+ 22Rvi PTEN shRNA-2

Figure 1. PTEN knockdown by siRNAs promotes glucose consumption and lactate production. (A) The siRNA sequences used in this experiment. (B) DU-145 and 22Rv1 cells were transfected with $40 \mathrm{nM}$ control siRNA and indicated concentrations of two different siRNAs targeting PTEN (PTEN siRNA-1 and PTEN siRNA-2) for $48 \mathrm{~h}$, after which samples were collected for quantitative real-time PCR and (C) Western blotting with antibodies against PTEN and $\beta$-actin. (D) DU-145 and 22Rv1 cells were transfected with $40 \mathrm{nM}$ control siRNA and PTEN siRNAs (PTEN siRNA-1 and PTEN siRNA-2) for 48 h, after which samples were collected for Western blotting with antibodies against PTEN, phospho-Akt (Ser473), Akt and $\beta$-actin. (E) Glucose consumption and lactate production was assessed in DU-145 cells and 22Rv1 cells which were transfected with 40 nM control siRNA and PTEN siRNAs (PTEN siRNA-1 and PTEN siRNA-2) for $48 \mathrm{~h}$. (F) The expression of catalytic enzymes in glycometabolism was detected by western blotting in DU-145 cells and 22Rv1 cells which were transfected with $40 \mathrm{nM}$ control siRNA and PTEN siRNAs (PTEN siRNA-1 and PTEN siRNA-2) for 48 h. (G) DU-145 and 22Rv1 cells which were infected with lentivirus containing PTEN shRNA or scramble shRNA, then cell viability was analyzed by MTT assay. HK1, hexokinase 1; HK2, hexokinase 2; PKM1/2, pyruvate kinase isozymes M1/M2; PKM2, pyruvate kinase isozyme M2; LDHA, lactate dehydrogenase A; PDH, pyruvate dehydrogenase; PFKP, phosphofructokinase platelet type; $\mathrm{PC}$, pyruvate carboxylase; $\mathrm{PTEN}$, gene of phosphate and tension homology deleted on chromosome ten. ${ }^{*} \mathrm{P}<0.05,{ }^{* *} \mathrm{P}<0.01$ compared with the cells transfected with control siRNA. 
$48 \mathrm{~h}$, the endogenous PTEN expression level was detected by quantitative real-time PCR and western blotting. The results revealed that endogenous PTEN was significantly decreased in 22Rv1 and DU-145 cells transfected with $40 \mathrm{nM}$ PTEN siRNAs compared with the control siRNAs (Fig. 1B and C). Furthermore, the phosphorylation of Akt at Serine 473 was significantly increased in DU-145 and 22Rv1 cells that were transfected with $40 \mathrm{nM}$ PTEN siRNAs after $48 \mathrm{~h}$ (Fig. 1D). Accompanying the activation of Akt, glucose consumption and lactate production was also significantly increased in PTEN knockdown DU-145 and 22Rv1 cells (Fig. 1E). Therefore, aerobic glycolysis was possibly increased in PTEN knockdown DU-145 and 22Rv1 cells. Furthermore, the expression of metabolic molecules related to glucose metabolism were detected and it was demonstrated that hexokinase HK2, the first key enzyme involved in glycolysis, was upregulated in PTEN knockdown DU-145 and 22Rv1 cells, especially in DU-145 cells (Fig. 1F). Upregulation of HK2 may be one of the contributors to the Warburg effect in PTEN knockdown DU-145 and 22Rv1 cells. Tumor metabolic reprogramming fuels the malignant growth and proliferation of cancer cells (13-15). Therefore, cell viability was increased in DU-145 cells that were infected with lentivirus containing PTEN shRNA compared with control shRNA (Fig. 1G). However, cell viability was not significantly altered in $22 \mathrm{Rv} 1$ cells that were infected with lentivirus containing PTEN shRNA. Although the PI3K/AKT/mTOR and RAF/MEK/ERK signaling pathways play an important role in prostate cancer progression, 22Rv1 prostate cancer cells have been revealed to be more dependent on the MEK/ERK pathway (16). Collectively, cell metabolism and cell viability was significantly altered in PTEN-loss DU-145 cells. Thus, metabolism reprogramming occurred, induced by PTEN deficiency in prostate cancer cells.

Intracellular metabolism reprogramming. High rates of loss of heterozygosity are observed at the $10 \mathrm{q} 23.3$ region containing the human PTEN gene in prostate cancer. The phenotypes of PTEN $^{+/-}$DU-145 cells are similar to prostate cancers with loss of heterozygosity in the PTEN gene (17-19). In addition, the cell viability induced by PTEN loss increased significantly in DU-145 cells. Therefore, we assessed the changes of key metabolites in PTEN knockdown DU-145 cells by metabolomics analysis. There are several metabolic pathway changes in tumor metabolism reprogramming, such as glycolysis, glutaminolysis, fatty acid de novo synthesis and fatty acid $\beta$-oxidation. Accordingly, the changes of key metabolites in these metabolic pathways were assessed in prostate cancer cells DU-145 transfected with PTEN siRNAs.

Glycolysis and glutaminolysis. Since glucose and glutamine are the main energy production and carbon sources for the malignant growth of tumor cells, we assessed the levels of metabolites in glycolysis and glutaminolysis. As revealed in Fig. 2, many organic acids involved in glycolysis and glutaminolysis were significantly increased, such as lactate, pyruvic acid, succinic acid, citric acid, fumaric acid, malic acid, and 2-ketoglutarate. In addition, the level of intracellular glutamine was decreased in PTEN knockdown DU-145 cells. Therefore, PTEN knockdown led to enhanced glycolysis and glutaminolysis in prostate cancer DU-145 cells.
Fatty acid de novo synthesis and $\beta$ oxidation. Unlike other epithelial tumors, primary prostate cancer relies on lipid metabolsim more than on aerobic glycolysis $(8,9)$. PTEN loss can lead to the overexpression of fatty acid synthase (FAS) and therefore facilitate cholesterol and fatty acid biosynthesis in prostate cancer (10). To investigate the function of PTEN in lipid metabolism, the metabolite changes in fatty acid de novo synthesis of PTEN knockdown DU-145 cells was analyzed. The results revealed that when PTEN expression was significantly decreased in prostate cancer cells, many fatty acylcarnitines involved in fatty acid de novo synthesis were enhanced, such as decanoyl-carnitine, dodecanoyl-carnitine, tetradecanoyl-carnitine, hexadecanoyl-carnitine, hexadecenoyl-carnitine, octadecanoyl-carnitine (Fig. 3). In parallel with enhanced fatty acid de novo synthesis, fatty acid $\beta$-oxidation was also increased in PTEN knockdown prostate cancer DU-145 cells. Compared with the control siRNA-transfected cells, many fatty acylcarnitines involved in fatty acid $\beta$-oxidation were significantly increased in DU-145 cells which were transfected with PTEN siRNAs, such as butanoyl-carnitine, hexanoyl-carnitine, hexenoyl-carnitine, hydroxyhexanoyl-carnitine, octenoyl-carnitine, decanoyl-carnitine, dodecanoyl-carnitine, tetradecanoyl-carnitine, hydroxytetradecanoyl-carnitine, hexadecanoyl-carnitine, hexadecenoyl-carnitine, and hydroxyhexadecanoyl-carnitine (Fig. 4). These results confirmed that PTEN loss can lead to the reprogramming of lipid metabolism in prostate cancer cells, including enhancement of fatty acid de novo synthesis and fatty acid $\beta$-oxidation, which provides bioenergy and biomolecules to fuel the malignant proliferation of cancer cells.

Branched-chain amino acids catabolism. The key enzyme that initiates the catabolism of branched-chain amino acids is branched-chain amino acid transaminase 1 (BCAT1), which is overexpressed in many types of cancers (20). Compared with healthy tissues, it has been reported that low levels of BCAT activity are present in all models of prostate cancer but the enzymatic levels are significantly altered in prostate cancer (21). Our results revealed that the products of branched-chain amino acid catabolism such as the levels of hydroxyisovaleryl-carnitine, methylbutanoyl-carnitine, and propanoyl-carnitine were increased in DU-145 cells which were transfected with PTEN siRNAs. Therefore, the catabolism of branched-chain amino acids was enhanced in PTEN-loss prostate cancer cell line DU-145 (Fig. 5). Whether the enhancement of BCAA catabolism is related to BCAT1 and the molecular mechanisms driving this still requires further investigation.

\section{Discussion}

Tumor suppressor gene PTEN deletion or mutation in combination with other genetic alterations can recapitulate the entire spectrum of human prostate cancer, from tumor initiation to metastasis. Metabolic reprogramming induced by PTEN loss is one of the key factors promoting prostate cancer tumorigenesis. The altered metabolism of cancer cells can provide abundant biomaterial and bioenergy for malignant proliferation and can confer a selective advantage for the survival and proliferation of cancer in the unique tumor microenvironment (22). Studies 

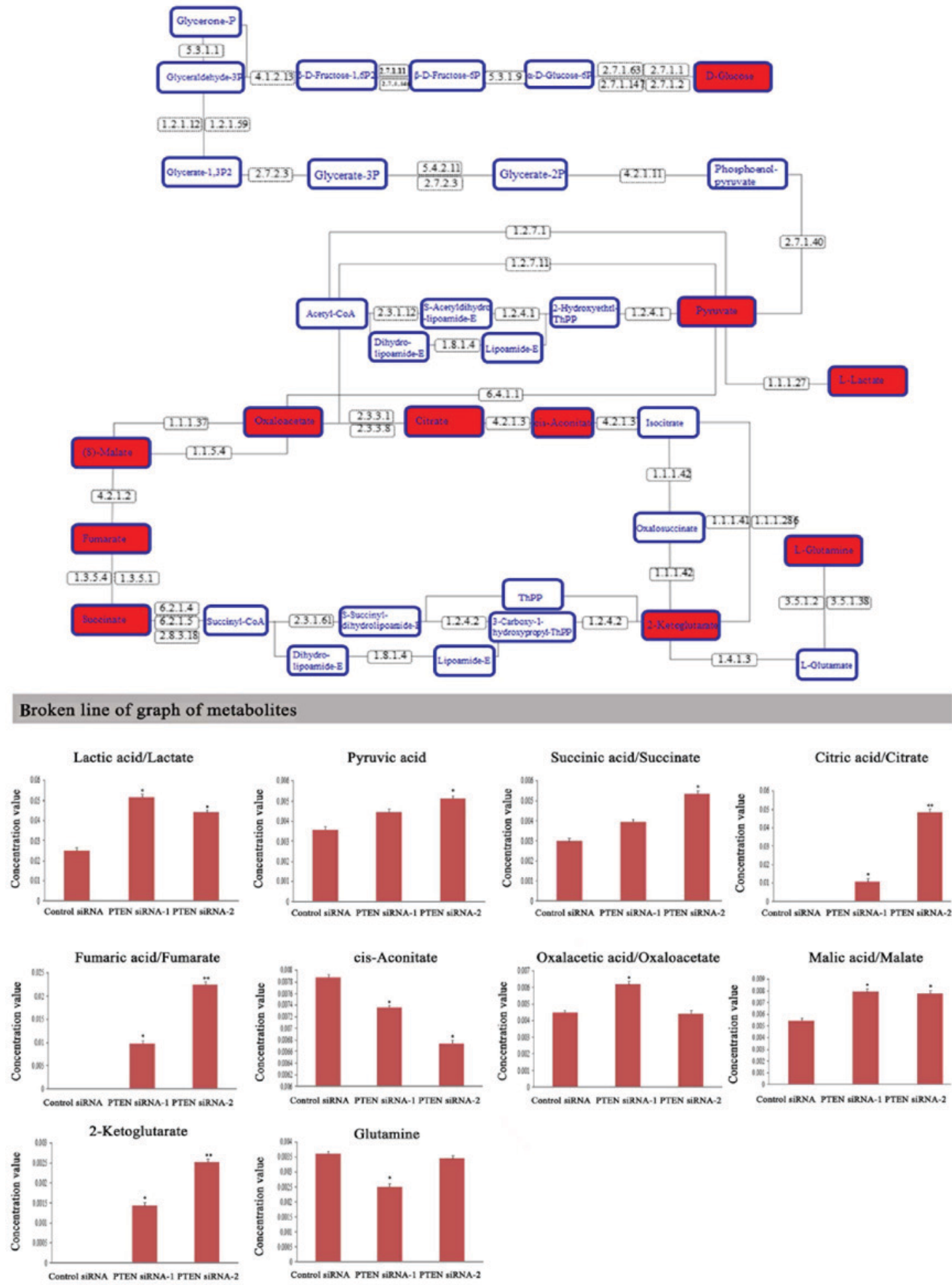

Figure 2. Metabolic profiling of intracellular organic acid involved in the glycolysis and glutaminolysis pathways. GC-MS data revealed the changes of organic acid levels in DU-145 cells which were transfected with PTEN siRNAs for $48 \mathrm{~h}$. The red-labeled metabolites in upper graph were significantly altered in the glycolysis and glutaminolysis pathways. 1.1.1.27, L-lactate dehydrogenase ( $\mathrm{LDH}, \mathrm{ldh}) ; 1.1 .1 .37$, malate dehydrogenase (MDH1); 1.1.1.42, isocitrate dehydrogenase (IDH1, IDH2); 1.1.5.4, malate dehydrogenase (mqo); 1.2.1.12, glyceraldehyde 3-phosphate dehydrogenase (GAPDH, gapA); 1.2.1.59, glyceraldehyde 3-phosphate dehydrogenase (NAD(P), gap2); 1.2.4.1, pyruvate dehydrogenase E1 component; 1.2.4.2, 2-oxoglutarate dehydrogenase E1 component; 1.2.7.1, pyruvate ferredoxin oxidoreductase alpha subunit (porA); 1.2.7.11, 2-oxoglutarate/2-oxoacid ferredoxin oxidoreductase subunit alpha (korA, oforA); 1.3.5.1, succinate dehydrogenase (ubiquinone) flavoprotein subunit; 1.3.5.4, fumarate reductase flavoprotein subunit; 1.4.1.3, glutamate dehydrogenase (NAD(P)+) (GLUD12, gdhA); 1.8.1.4, dihydrolipoamide dehydrogenase (DLD, lpd, pdhD); 2.3.1.12, pyruvate dehydrogenase E2 component (DLAT, aceF, pdhC); 2.3.1.61, 2-oxoglutarate dehydrogenase E2 component; 2.3.3.1, citrate synthase; 2.3.3.8, ATP citrate (pro-S)-lyase; 2.7.1.1, hexokinase (HK); 2.7.1.11, 6-phosphofructokinase 1(pfkA, PFK); 2.7.1.146, ADP-dependent phosphofructokinase/glucokinase (pfkC); 2.7.1.147, ADP-dependent glucokinase (ADPGK); 2.7.1.2, glucokinase (GCK); 2.7.1.40, pyruvate kinase (PK, pyk); 2.7.1.63 polyphosphate glucokinase (ppgK); 2.7.2.3, phosphoglycerate kinase (PGK, pgk); 2.8.3.18, succinyl-CoA:acetate CoA-transferase; 3.5.1.2 glutaminase (glsA, GLS), 3.5.1.38, glutamin-(asparagin-)ase (aspQ, ansB, ansA); 4.1.2.13, fructose-bisohosphate aldolase, class I (ALDO); 4.2.1.11, enolase (ENO, eno); 4.2.1.2, fumarate hydratase, class I; 4.2.1.3, aconitate hydratase; 5.3.1.1, triosephosphate isomerase (TIM) (TPI, tpiA); 5.3.1.9, glucose-6-phosphate isomerase (GPI, pgi); 5.4.2.11, 2,3-bisphosphoglycerate-dependent phosphoglycerate mutase (PGAM, pgmA); 5.4.2.11, 2,3-bisphosphoglycerate-independent phosphoglycerate mutase (pgmI); 6.2.1.4, succinyl-CoA synthetase alpha subunit (LSC1); 6.2.1.5, succinyl-CoA synthetase alpha subunit (sucD); 6.4.1.1, pyruvate carboxylase (PC, pyc). ${ }^{*} \mathrm{P}<0.05,{ }^{* *} \mathrm{P}<0.01$ vs. the cells transfected with control siRNA. 


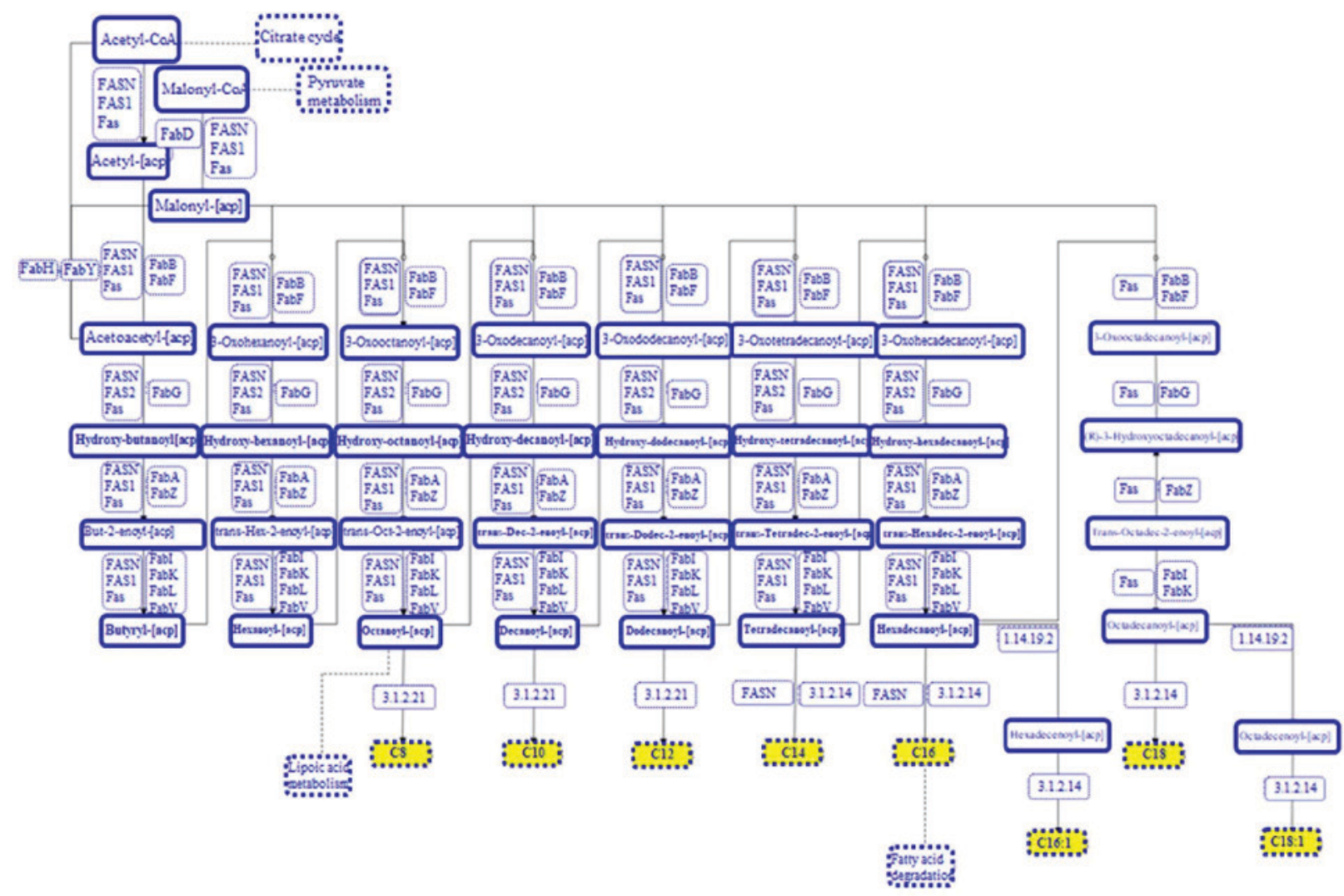

\section{Broken line of graph of metabolites}

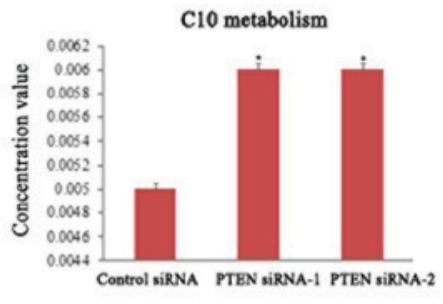

C16 metabolism

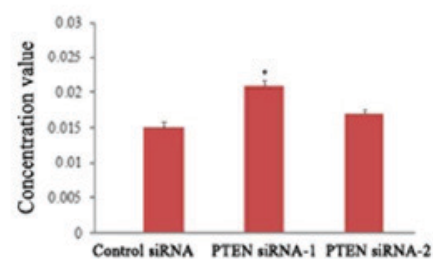

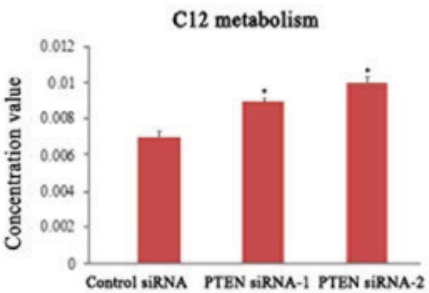

C16:1 metabolism

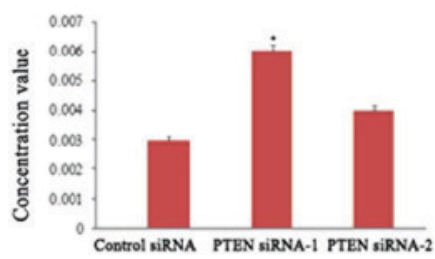

C14 metabolism

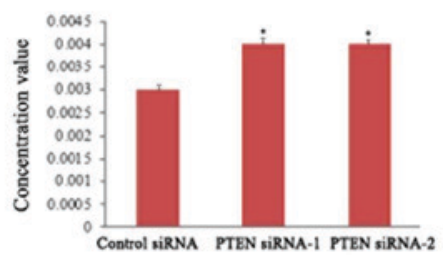

C18:1 metabolism

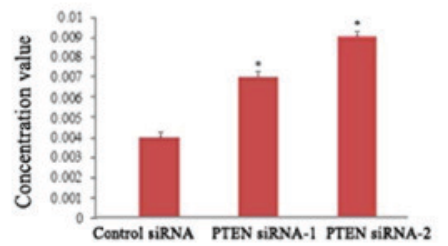

Figure 3. Metabolic profiling of intracellular metabolites involved in fatty acid de novo synthesis. LC-MS data revealed the changes of metabolite levels in DU-145 cells which were transfected with PTEN siRNAs for $48 \mathrm{~h}$. The yellow-labeled metabolites in upper graph were significantly altered in the fatty acid de novo synthesis pathway. 1.14.19.2, acyl-[acyl-carrier-protein] desaturase; 3.1.2.14, fatty acyl-ACP thioesterase B; 3.1.2.21, medium-chain acyl-[acyl-carrier-protein] hydrolase; FabA, 3-hydroxyacyl-[acyl-carrier-protein] dehydratase; FabB, 3-oxoacyl-[acyl-carrier-protein] synthase I; FabD, [acyl-carrier-protein] S-malonyltransferase; FabF, 3-oxoacyl-[acyl-carrier-protein] synthase II; FabG, 3-oxoacyl-[acyl-carrier-protein] reductase; FabH, 3-oxoacyl-[acyl-carrier-pro tein] synthase III; FabI, enoyl-[acyl-carrier-protein] reductase I; FabK, enoyl-[acyl-carrier-protein] reductase II; FabL, enoyl-[acyl-carrier-protein] reductase III; FabV, enoyl-[acyl-carrier-protein] reductase/trans-2-enoyl-CoA reductase (NAD+); FabY, acetoacetyl-[acyl-carrier-protein] synthase; FabZ, 3-hydroxyacyl-[acyl-carrier-protein] dehydratase; Fas, fatty acid synthase, bacteria type; FAS1, fatty acid synthase subunit beta, fungi type; FAS2, fatty acid synthase subunit alpha, fungi type; FASN,fatty acid synthase, animal type; C10, decanoyl-carnitine; C12, dodecanoyl-carnitine; C14, tetradecanoyl-carnitine; C16, hexadecanoyl-carnitine; C16:1, hexadecenoyl-carnitine; C18:1, octadecanoyl-carnitine. "P<0.05 vs. cells transfected with control siRNA.

have demonstrated that oncogenes and tumor suppressor genes can drive metabolic reprogramming by regulating the expression or activity of metabolic enzymes (13). It has been reported that loss of PTEN promoted aerobic glycolysis in prostate epithelial cells (17). In addition, cells derived from PTEN transgenic mice revealed reduced glucose and glutamine uptake and increased mitochondrial oxidative phosphorylation (11). Compared with previous studies, we demonstrated that loss of PTEN in prostate cancer cells resulted in enhanced fatty acid de novo synthesis and $\beta$-oxidation, 

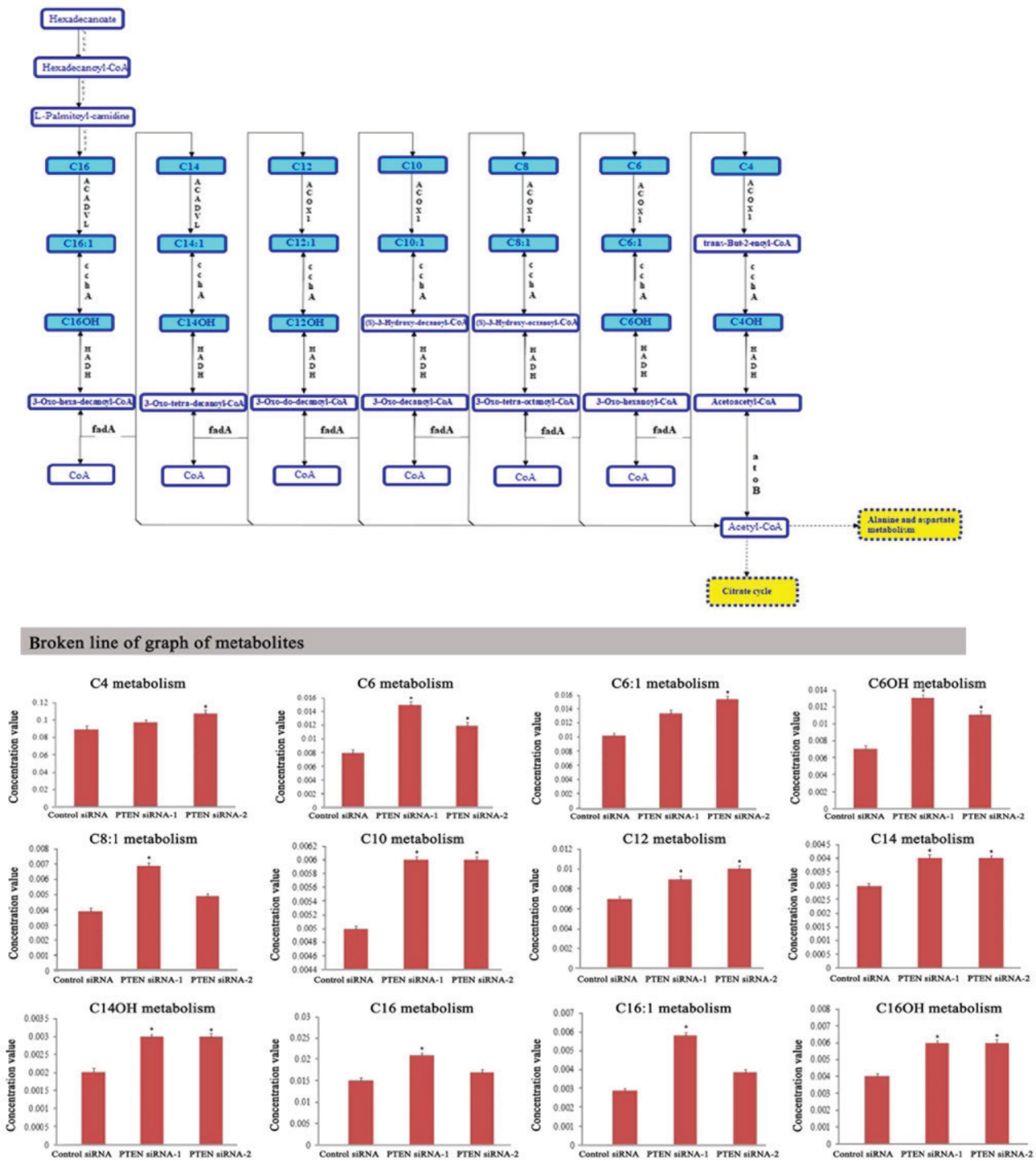

Figure 4. Metabolic profiling of intracellular metabolites involved in fatty acid $\beta$-oxidation. LC-MS data revealed the changes of metabolite levels in DU-145 cells which were transfected with PTEN siRNAs for $48 \mathrm{~h}$. The blue-labeled metabolites in upper graph were significantly altered in fatty acid the $\beta$-oxidation pathway. ACADVL, very long chain acyl-CoA dehydrogenase; ACOX1, acyl-CoA oxidase; ACSL, long-chain acyl-CoA synthetase; atoB, acetyl-CoA C-acetyltransferase; CPT1, carnitine O-palmitoyltransferase 1; CPT2, carnitine O-palmitoyltransferase 2; echA, enoyl-CoA hydratase; fadA, acetyl-CoA acyltransferase; HADH, 3-hydroxyacyl-CoA dehydrogenase; HIBCH, 3-hydroxyisobutyryl-CoA hydrolase; IVD, isovaleryl-CoA dehydrogenase; mmsA, malonate-semialdehyde dehydrogenase (acetylating); mmsB, 3-hydroxyisobutyrate dehydrogenase vdh valine dehydrogenase; vorA, 2-oxoisovalerate ferredoxin oxidoreductase, alpha subunit; $\mathrm{C} 4$, Butanoyl-carnitine; $\mathrm{C} 6$, hexanoyl-carnitine; C6:1, hexenoyl-carnitine; $\mathrm{C} 6 \mathrm{OH}$, hydroxyhexanoyl-carnitine; C8:1, octenoyl-carnitine; C10, decanoyl-carnitine; C12, dodecanoyl-carnitine; C14, tetradecanoyl-carnitine; C14OH, hydroxytetradecanoyl-carnitine; C16, hexadecanoyl-carnitine; $\mathrm{C} 16: 1$, hexadecenoyl-carnitine; $\mathrm{C} 16 \mathrm{OH}$, hydroxyhexadecanoyl-carnitine. ${ }^{\mathrm{P}}<0.05$ vs. cells transfected with control siRNA.

and enhanced branched chain amino acid catabolism in addition to enhanced glycolysis and glutaminolysis. Thus, these metabolic characteristics may be new targets for prostate cancer therapy.
The Warburg effect is one of the metabolic characteristics of cancer cells, which exhibits high rates of glycolysis with increased glucose consumption and lactate production (7). Many metabolic molecules are involved in the Warburg 


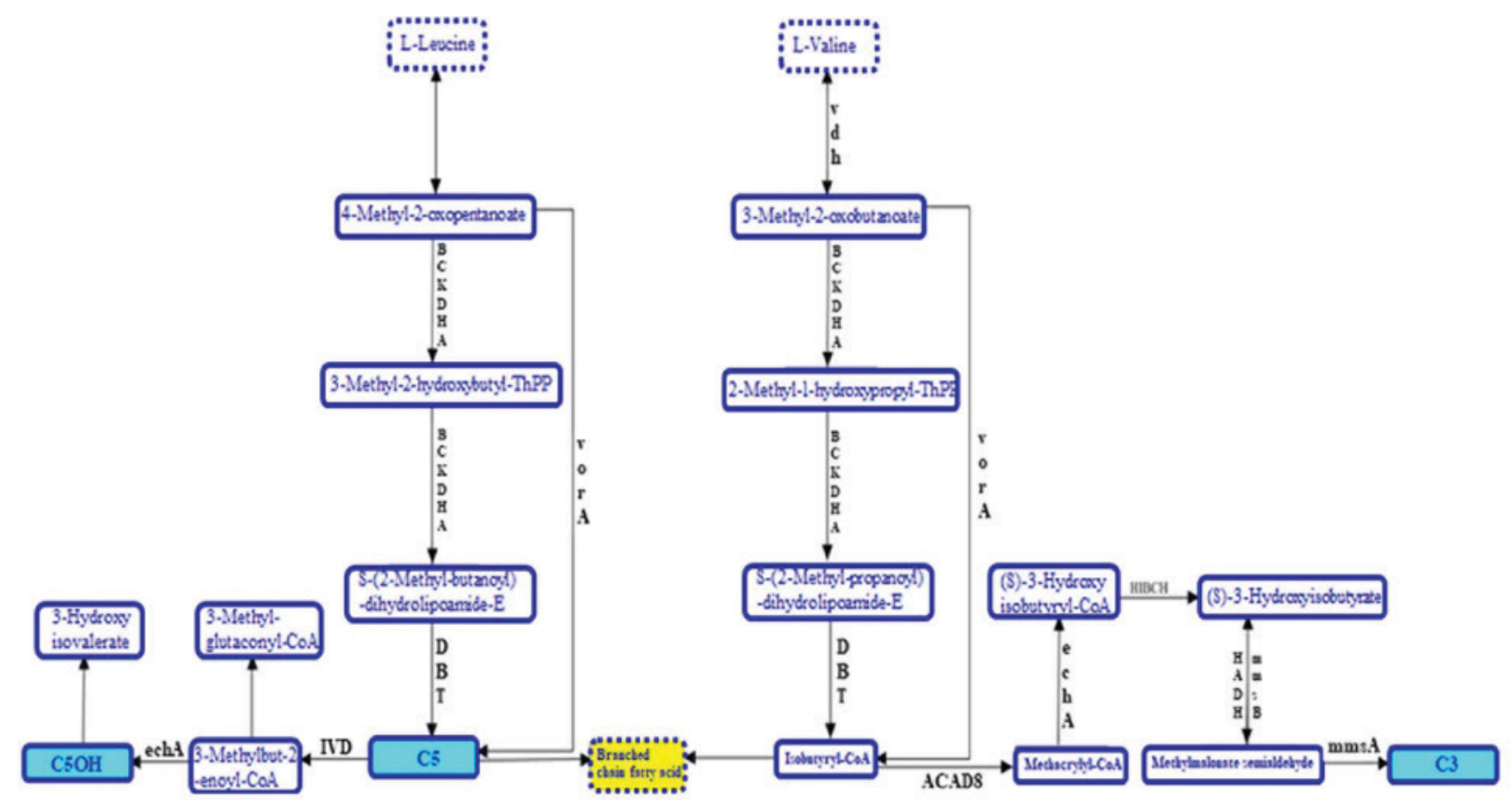

Broken line of graph of metabolites
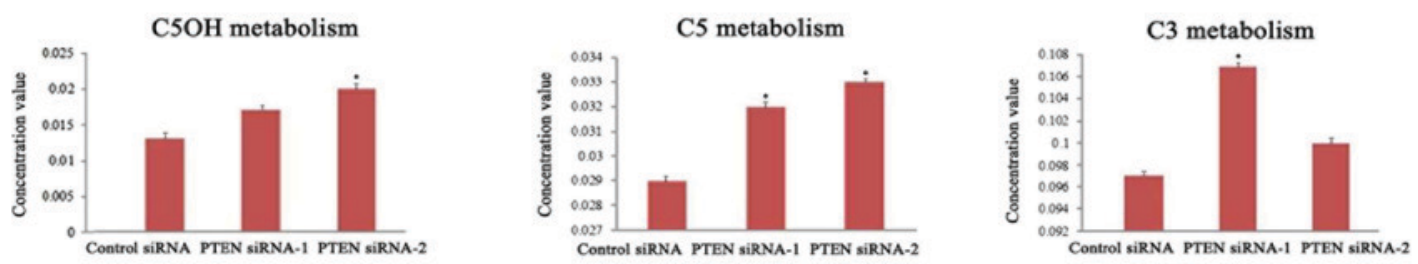

Figure 5. Metabolic profiling of intracellular metabolites involved in branched-chain amino acid catabolism. LC-MS data revealed the changes of metabolite levels in DU-145 cells which were transfected with PTEN siRNAs for $48 \mathrm{~h}$. The blue-labeled metabolites in upper graph were significantly altered in the branched-chain amino acid catabolism pathway. ACAD8, isobutyryl-CoA dehydrogenase; BCKDHA, 2-oxoisovalerate dehydrogenase E1 component alpha subunit; DBT, 2-oxoisovalerate dehydrogenase E2 component (dihydrolipoyl transacylase); echA, enoyl-CoA hydratase; HADH, 3-hydroxyacyl-CoA dehydrogenase; HIBCH, 3-hydroxyisobutyryl-CoA hydrolase; IVD, isovaleryl-CoA dehydrogenase; mmsA, malonate-semialdehyde dehydrogenase (acetylating); $\mathrm{mmsB}, 3$-hydroxyisobutyrate dehydrogenase; vdh, valine dehydrogenase ( $\left.\mathrm{NAD}^{+}\right)$; vorA, 2-oxoisovalerate ferredoxin oxidoreductase, alpha subunit; $\mathrm{C} 5 \mathrm{OH}$, hydroxyisovaleryl-carnitine; $\mathrm{C}$, methylbutanoyl-carnitine; $\mathrm{C} 3$, propanoyl-carnitine. ${ }^{*} \mathrm{P}<0.05$ vs. cells transfected with control siRNA.

effect of PTEN-deficiency-driven prostate tumorigenesis. Firstly, hexokinase HK2, which catalyzes the essentially irreversible first step of the glycolytic pathway, mediated the Warburg effect and malignant growth of PTEN and p53 deficiency-driven prostate cancers. PTEN loss increased HK2 mRNA translation through AKT/mTORC1/4EBP1 signaling (17). Secondly, fructose 2,6-bisphosphate (F2,6BP), the most potent allosteric activator of the glycolytic enzyme phosphofructokinase-1 (PFK-1), promoted the Warburg effect in PTEN-deficient cells. PTEN loss negatively affected the activity of the E3 ligase APC/C-Cdh1, resulting in the stabilization of the enzyme PFKFB3 and increased the synthesis of its product $\mathrm{F} 2,6 \mathrm{BP}_{2}(23)$. Thirdly, PTEN loss resulted in the activation of the PI3K/Akt pathway, which contributed to the phosphorylation of the enzyme PFKFB2 and activation of the glycolytic enzyme phosphofructokinase-2 (PFK-2) in prostate cancer LNCaP cells (24). Finally, Akt activation induced by PTEN deficiency promoted the expression of GluT1 on the plasmamembrane and glycolysis in cancer cells (25). Therefore, PTEN loss enhanced glucose uptake and glycolytic enzymatic activity, which promoted the glycolysis pathway in cancer cells.
Cancer cells utilize glucose and glutamine as primary carbon sources to feed mitochondrial intermediates for biosynthetic precursors. The enhancement of the carbon flux through glycolysis and glutaminolysis fulfills the energetic and biosynthetic demands of cancer cells. In addition to glycolysis, glutamonolysis is also enhanced in PTEN knockdown prostate cancer cells. PTEN can reduce the protein level of glutaminase (GLS) which is the first rate-limiting enzyme in the glutaminolysis pathway. PTEN can promote the activity of the E3 ubiquitin ligase anaphase-promoting complex/cyclosome-Cdh1 (APC/C-Cdh1), which targets GLS for degradation (11). Loss of PTEN stabilized glutaminase and promoted glutaminolysis in prostate cancer cells.

Lipid metabolism reprogramming is a major contributor to sustaining prostate cancer development (9). This lipid metabolism dysregulation is a prominent feature that encompasses elevated de novo lipogenesis including steroid-hormone biosynthesis as well as $\beta$-oxidation of fatty acids (26). Deficiency of PTEN and activation of PI3K/Akt can activate the transcriptional factor SREBP1C, which in turn transcribes genes involved in fatty acid and cholesterol biosynthesis (27). PTEN inhibits the synthesis of long-chain 
saturated fatty acids by inhibiting the expression of fatty acid synthase (FAS) in a lipid phosphatase-dependent manner (10). PTEN deficiency was revealed to play an important role in the overexpression of the FAS protein and the enhancement of fatty acid de novo synthesis in prostate cancer cells (10). It has been reported that lipid production in prostate-specific conditional $\mathrm{PTEN}^{-/}$mice was enhanced compared with wild-type mice. Furthermore, ATP-citrate lyase (ACLY), the first rate-limiting enzyme involved in de novo lipogenesis, was also revealed to be enhanced in prostate-specific conditional $\mathrm{PTEN}^{-/}$mice compared with wild-type mice (28). In addition, inactivation of pyruvate dehydrogenase (PDHA1) can inhibit prostate cancer development and block enhancement of lipid biosynthesis induced by PTEN loss (28). Accordingly, our research also discovered that fatty acid de novo synthesis was increased in PTEN knockdown prostate cancer cells. Therefore, loss of PTEN in prostate cancer cells resulted in enhancement of fatty acid de novo synthesis and fatty acid $\beta$-oxidation.

The branched-chain amino acids (BCAAs) leucine, isoleucine, and valine can be used for protein synthesis or oxidized as a tumor energy supply. Branched-chain aminotransferase 1 (BCAT1), the enzyme involved in the first step of BCAA catabolism, contributes to the metabolic reprogramming and malignant proliferation of cancer cells (20). It has been reported that BCAT1 is a target gene of c-Myc and induces the cell proliferation of nasopharyngeal carcinoma (29). In addition, microRNA-218 negatively regulated BCAT1 and inhibited the growth of prostate cancer (30). Our research revealed that the catabolism of branched-chain amino acids was upregulated in PTEN-knockdown prostate cancer cells. Whether the enhancement of BCAA catabolism is related to BCAT1 or related to other molecular mechanisms still requires further investigation.

In conclusion, in the present study, using metabolomics analysis it was revealed that PTEN deficiency promoted metabolic reprogramming by enhancing glycolysis, glutaminolysis, fatty acid synthesis and $\beta$-oxidation, and branched-chain amino acid catabolism in prostate cancer cells. The metabolic reprogramming induced by PTEN loss provided biomaterials and bioenergy for the proliferation of prostate cancer cells. This study implicates a potential therapeutic target for prostate cancer cells with PTEN mutation or deletion.

\section{Acknowledgements}

Not applicable.

\section{Funding}

This study was supported by Cancer Biology State Key Laboratory (grant no. CBSKL2014Z09). This study was supported by the National Natural Science Foundation of China (grant nos. 81672542, 81572504 and 81772745); the Natural Science Foundation of Shaanxi Province (grant no. 2017SF-187).

\section{Availability of data and materials}

All data generated or analyzed during the present study are included in this published article.

\section{Authors' contributions}

$\mathrm{XZ}$ and $\mathrm{XY}$ contributed to the research conception and design. $\mathrm{XX}, \mathrm{JW}$ and XS contributed to the data analysis and interpretation. XAL, YG and XL contributed to the statistical analysis. LS designed the study and wrote the manuscript. LY and HW reviewed and edited the manuscript and were also involved in the conception of the study. All authors approved the final manuscript.

\section{Ethics approval and consent to participate}

Not applicable.

\section{Patient consent for publication}

Not applicable.

\section{Competing interests}

The authors declare that they have no competing interests.

\section{References}

1. Li J, Yen C, Liaw D, Podsypanina K, Bose S, Wang SI, Puc J, Miliaresis C, Rodgers L, McCombie R, et al: PTEN, a putative protein tyrosine phosphatase gene mutated in human brain, breast, and prostate cancer. Science 275: 1943-1947, 1997.

2. Wang S, Gao J,LeiQ, Rozengurt N,Pritchard C, Jiao J, Thomas GV, Li G, Roy-Burman P, Nelson PS, et al: Prostate-specific deletion of the murine Pten tumor suppressor gene leads to metastatic prostate cancer. Cancer Cell 4: 209-221, 2003.

3. Majumder PK, Yeh JJ, George DJ, Febbo PG, Kum J, Xue Q, Bikoff R, Ma H, Kantoff PW, Golub TR, et al: Prostate intraepithelial neoplasia induced by prostate restricted Akt activation: the MPAKT model. Proc Natl Acad Sci USA 100: 7841-7846, 2003.

4. Chang CJ, Mulholland DJ, Valamehr B, Mosessian S, Sellers WR and Wu H: PTEN nuclear localization is regulated by oxidative stress and mediates p53-dependent tumor suppression. Mol Cell Biol 28: 3281-3289, 2008.

5. Freeman DJ, Li AG, Wei G, Li HH, Kertesz N, Lesche R, Whale AD, Martinez-Diaz H, Rozengurt N, Cardiff RD, et al: PTEN tumor suppressor regulates p53 protein levels and activity through phosphatase-dependent and -independent mechanisms. Cancer Cell 3: 117-130, 2003.

6. Vivanco I, Palaskas N, Tran C, Finn SP, Getz G, Kennedy NJ, Jiao J, Rose J, Xie W, Loda M, et al: Identification of the JNK signaling pathway as a functional target of the tumor suppressor PTEN. Cancer Cell 11: 555-569, 2007.

7. Warburg O: On respiratory impairment in cancer cells. Science 124: 269-270, 1956.

8. Schlaepfer IR, Rider L, Rodrigues LU, Gijón MA, Pac CT, Romero L, Cimic A, Sirintrapun SJ, Glodé LM, Eckel RH and Cramer SD: Lipid catabolism via CPT1 as a therapeutic target for prostate cancer. Mol Cancer Ther 13: 2361-2371, 2014.

9. Wu X, Daniels G, Lee P and Monaco ME: Lipid metabolism in prostate cancer. Am J Clin Exp Urol 2: 111-120, 2014.

10. Van de Sande T, De Schrijver E, Heyns W, Verhoeven G and Swinnen JV: Role of the phosphatidylinositol 3'-kinase/PTEN/Akt kinase pathway in the overexpression of fatty acid synthase in LNCaP prostate cancer cells. Cancer Res 62: 642-646, 2002.

11. Garcia-Cao I, Song MS, Hobbs RM, Laurent G, Giorgi C, de Boer VC, Anastasiou D, Ito K, Sasaki AT, Rameh L, et al: Systemic elevation of PTEN induces a tumor-suppressive metabolic state. Cell 149: 49-62, 2012.

12. Livak KJ and Schmittgen TD: Analysis of relative gene expression data using real-time quantitative PCR and the 2(-Delta Delta C(T)) method. Methods 25: 402-408, 2001.

13. Hsu PP and Sabatini DM: Cancer cell metabolism: Warburg and beyond. Cell 134: 703-707, 2008.

14. Kroemer G and Pouyssegur J: Tumor cell metabolism: Cancer's Achilles' heel. Cancer Cell 13: 472-482, 2008. 
15. Shen L, Sun X, Fu Z, Yang G, Li J and Yao L: The fundamental role of the p53 pathway in tumor metabolism and its implication in tumor therapy. Clin Cancer Res 18: 1561-1567, 2012.

16. Toren P, Kim S, Johnson F and Zoubeidi A: Combined AKT and MEK pathway blockade in pre-clinical models of enzalutamide-resistant prostate cancer. PLoS One 11: e0152861, 2016.

17. Wang L, Xiong H, Wu F, Zhang Y, Wang J, Zhao L, Guo X, Chang LJ, Zhang Y, You MJ, et al: Hexokinase 2-mediated Warburg effect is required for PTEN- and p53-deficiency-driven prostate cancer growth. Cell Rep 8: 1461-1474, 2014

18. Kwabi-Addo B, Giri D, Schmidt K, Podsypanina K, Parsons R, Greenberg N and Ittmann M: Haploinsufficiency of the Pten tumor suppressor gene promotes prostate cancer progression. Proc Natl Acad Sci USA 98: 11563-11568, 2001.

19. Fraser M, Zhao H, Luoto KR, Lundin C, Coackley C, Chan N Joshua AM, Bismar TA, Evans A, Helleday T and Bristow RG: PTEN deletion in prostate cancer cells does not associate with loss of RAD51 function: Implications for radiotherapy and chemotherapy. Clin Cancer Res 18: 1015-1027, 2012.

20. Tönjes M, Barbus S, Park YJ, Wang W, Schlotter M, Lindroth AM, Pleier SV, Bai AHC, Karra D, Piro RM, et al: BCAT1 promotes cell proliferation through amino acid catabolism in gliomas carrying wild-type IDH1. Nat Med 19: 901-908, 2013.

21. Billingsley KL, Park JM, Josan S, Hurd R, Mayer D, Spielman-Sun E, Nishimura DG, Brooks JD and Spielman D: The feasibility of assessing branched-chain amino acid metabolism in cellular models of prostate cancer with hyperpolarized [1-(13) C]-ketoisocaproate. Magn Reson Imaging 32: 791-795, 2014.

22. Yue S, Li J, Lee SY, Lee HJ, Shao T, Song B, Cheng L, Masterson TA, Liu X, Ratliff TL and Cheng JX: Cholesteryl ester accumulation induced by PTEN loss and PI3K/AKT activation underlies human prostate cancer aggressiveness. Cell Metab 19: 393-406, 2014.

23. Cordero-Espinoza L and Hagen T: Increased concentrations of fructose 2,6-bisphosphate contribute to the Warburg effect in phosphatase and tensin homolog (PTEN)-deficient cells. J Biol Chem 288: 36020-36028, 2013.
24. Moon JS, Jin WJ, Kwak JH, Kim HJ, Yun MJ, Kim JW, Park SW and Kim KS: Androgen stimulates glycolysis for de novo lipid synthesis by increasing the activities of hexokinase 2 and 6-phosphofructo-2-kinase/fructose-2,6-bisphosphatase 2 in prostate cancer cells. Biochem J 433: 225-233, 2011.

25. Phadngam S, Castiglioni A, Ferraresi A, Morani F, Follo C and Isidoro C: PTEN dephosphorylates AKT to prevent the expression of GLUT1 on plasmamembrane and to limit glucose consumption in cancer cells. Oncotarget 7: 84999-85020, 2016.

26. Butler LM, Centenera MM and Swinnen JV: Androgen control of lipid metabolism in prostate cancer: Novel insights and future applications. Endocr Relat Cancer 23: R219-R227, 2016.

27. Horie Y, Suzuki A, Kataoka E, Sasaki T, Hamada K, Sasaki J, Mizuno K, Hasegawa G, Kishimoto H, Iizuka M, et al: Hepatocyte-specific Pten deficiency results in steatohepatitis and hepatocellular carcinomas. J Clin Invest 113: 1774-1783, 2004.

28. Chen J, Guccini I, Di Mitri D, Brina D, Revandkar A, Sarti M, Pasquini E, Alajati A,Pinton S, Losa M,et al: Compartmentalized activities of the pyruvate dehydrogenase complex sustain lipogenesis in prostate cancer. Nat Genet 50: 219-228, 2018

29. Zhou W, Feng X, Ren C, Jiang X, Liu W, Huang W, Liu Z, Li Z, Zeng L, Wang L, et al: Over-expression of BCAT1, a c-Myc target gene, induces cell proliferation, migration and invasion in nasopharyngeal carcinoma. Mol Cancer 12: 53, 2013.

30. Zhu W, Shao Y and Peng Y: MicroRNA-218 inhibits tumor growth and increases chemosensitivity to CDDP treatment by targeting BCAT1 in prostate cancer. Mol Carcinog 56: 1570-1577, 2017.

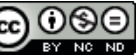

This work is licensed under a Creative Commons Attribution-NonCommercial-NoDerivatives 4.0 International (CC BY-NC-ND 4.0) License. 